\title{
Evaluación del crecimiento en cabritos hipotiroideos en un rodeo de la Provincia de Formosa, Argentina*
}

\author{
Ortiz, M.L. ${ }^{1}$; Brem, J.J. ${ }^{1}$ Mancebo, O.A. ${ }^{2}$; Trulls, H.E. ${ }^{1}$; Brem, J.C. ${ }^{1}$ \\ ${ }^{1}$ Laboratorio de Análisis Físicoquímicos anexo a la Cátedra de Biofísica, Facultad de Ciencias Veterinarias, UNNE, \\ Sargento Cabral 2139, Corrientes (3400), Argentina, Tel 03783-430101. E-mail: jjbrem@vet.unne.edu.ar. \\ ${ }^{2}$ Centro de Diagnóstico e Investigaciones Veterinarias de Formosa (CEDIVEF), Formosa, Argentina.
}

\begin{abstract}
Resumen
Ortiz, M.L.; Brem, J.J.; Mancebo, O.A.; Trulls, H.E.; Brem, J.C.: Evaluación del crecimiento en cabritos hipotiroideos en un rodeo de la Provincia de Formosa, Argentina. Rev. vet. 21: 2, 99-105, 2010. Las hormonas tiroideas regulan el metabolismo intermedio del animal y el bocio es el agrandamiento de la glándula tiroides que aparece como mecanismo compensatorio en respuesta al estado de hipofunción. Bajos niveles hormonales estimulan la liberación de tirotrofina hipofisaria llevando a la hiperplasia glandular característica. En adultos esta patología provoca principalmente trastornos reproductivos y en animales jóvenes un deficiente crecimiento y desarrollo. Con el objeto de cuantificar el efecto sobre estos últimos aspectos y correlacionar los niveles de hormonas tiroideas con la evolución de parámetros morfoestructurales, luego de ser confirmada la funcionalidad glandular a través de dosajes hormonales, se desarrolló el presente ensayo determinando peso vivo, condición corporal y medidas morfoestructurales: alzada a la cruz, diámetro longitudinal, perímetro torácico, diámetro dorsoesternal, ancho de grupa y perímetro de caña del miembro anterior. Combinando estos valores se obtuvieron diferentes índices: corporal, de proporcionalidad (IPRO), metacarpotorácico, de profundidad relativa de tórax (IPRP) y pelviano transversal (IPET) en cabritos de ambos sexos y de tres meses de edad, recientemente destetados. Se trabajó con dos lotes de diez animales, uno con claros síntomas de bocio y el otro sin evidencias clínicas (grupo control). Se realizaron cuatro mediciones mensuales sucesivas de las variables dependientes, utilizando un diseño de medidas repetidas completo al azar. No se hallaron diferencias significativas en la evolución del peso vivo y la condición corporal. En cuanto a las medidas morfométricas, hubo diferencias significativas $(p<0,05)$ en los valores de alzada a la cruz, que resultaron ser menores en los animales hipotiroideos, y en consecuencia los índices IPRO, IPRP e IPET resultaron significativamente mayores. En el análisis de correlación entre esta variable y el estado hipo o eutiroideo, se obtuvo un coeficiente de 0,70 con alto grado de significancia $(\mathrm{p}=0,01)$; las otras variables morfométricas no mostraron diferencias significativas.
\end{abstract}

Palabras clave: cabra, hipotiroidismo, crecimiento, variables morfométricas.

\begin{abstract}
Ortiz, M.L.; Brem, J.J.; Mancebo, O.A.; Trulls, H.E.; Brem, J.C.: Evaluation of growth in small goats with hypothyroidism from a herd of Formosa, north-east Argentina. Rev. vet. 21: 2, 99-105, 2010. Thyroid hormones control the intermediate animal metabolism. Goitre is an enlargement of the gland caused, in hypothyroid states, by a negative feedback mechanism. Low levels of thyroid hormones increase the release of thyrotropin, which stimulates the thyroid gland leading to hyperplasia. This pathology alters different organic systems, mainly affecting reproduction as well as growth and development in young animals. The objectives of this work were to quantify these effects and to correlate the hormone levels with the evolution of morphological parameters, indicators of growth and development, after confirmation of goiter by determination of hormone levels. Three months-old, both sexes goats from Anglo Nubia, Criolla and Boer breeds, from a herd of Ibarreta (Formosa, Argentina), were used. Ten animals had goitre signs while other ten were clinically healthy. Monthly, parameters such as body weight, corporal condition and morphometric measures were determined, considering: wither height, longitudinal diameter, thoracic perimeter, dorsoesternal diameter, rump width and metacarpal bone diameter. These parameters allowed to
\end{abstract}


obtain the following indexes: corporal, proportionality (IPRO), metacarpal-thoracic, relative depth of thorax (IPRP) and cross-section of pelvis (IPET). Data were analyzed according to a completely randomized design with repeated measures. There were no significant differences in the evolution of body weight and corporal condition, but there were lower values ( $\mathrm{p}$ $<0.05$ ) for wither height in hypothyroid goats, so IPRO, IPRP and IPET indexes were higher. The analysis of the correlation of this variable with the level of hormones shows a coefficient of 0.70 , with high degree of significance $(p=0.01)$. The other variables did not show significant differences.

Key words: goat, hypothyroidism, growth, morphometric variables.

\section{INTRODUCCIÓN}

La cría y explotación de cabras es una actividad orientada a la producción de proteínas de alto valor biológico de su carne y leche. Además, es una importante fuente de ingreso familiar de criadores minifundistas, ofreciendo una serie de ventajas como ser baja inversión de capital, necesidad de espacios reducidos, alta capacidad reproductiva y facilidad de manejo ${ }^{13}$. Es un recurso muy interesante por la diversidad de productos que brinda al hombre, presentando notables ganancias económicas por su bajo costo de mantenimiento, generando manufacturas de elevada demanda y precio, ocupación estable y, con un adecuado manejo, permite un sistema sustentable y ecológicamente equilibrado ${ }^{5}$.

La producción caprina en el centro-oeste de Chaco y Formosa (Argentina) se realiza en forma extensiva sobre pastizales naturales (pajonales, arbustos y montes, o combinados), existiendo una oferta forrajera muy variada en cuanto a cantidad y calidad, no sólo estacional sino también entre localidades y aún dentro de un mismo potrero.

La caracterización morfológica del caprino es considerada una herramienta válida que aporta información para la definición de parámetros morfofuncionales, permitiendo realizar predicciones de crecimiento y producción de carne ${ }^{29}$. Las variables zoométricas pueden ser consideradas en la caracterización racial (de hecho forman parte del tipo o estándar de razas), pero existe un fuerte aporte del componente ambiental para su expresión ${ }^{11}$. En esta caracterización es importante determinar si mediante procedimientos discriminatorios se pueden diferenciar individuos dentro de una misma población base.

En el bovino lechero, las hormonas tiroideas, además de las conocidas funciones sobre el mantenimiento del metabolismo basal y equilibrio de los sistemas orgánicos, son constituyentes claves de los fenómenos de crecimiento, desarrollo y reproducción, y sus niveles se ven afectados por múltiples factores entre los que se cuentan la edad, balance energético y momento del ciclo reproductivo y de lactancia, lo mismo que el nivel de producción y de las patologías primarias que puedan afectar directamente a la glándula tiroides ${ }^{22}$.

En animales en crecimiento, la dinámica del desarrollo óseo y la ganancia de peso incrementan los re- querimientos energéticos, elevando la tasa metabólica y generando un mayor consumo de oxígeno. La estricta regulación metabólica que demanda el crecimiento estimula las vías anabólicas a nivel celular mediante acciones permisivas de tipo endocrino. Como las hormonas tiroideas controlan la actividad mitocondrial de los mamíferos, existe una alta dependencia de la regulación glandular para garantizar el aporte de ATP a estos procesos anabólicos ${ }^{20}$.

Algunos autores establecieron que la deficiencia de hormonas tiroideas en animales jóvenes provoca un retraso en el crecimiento e inmadurez ósea, destacándose que la acción de la triiodotironina sobre el crecimiento lineal, desarrollo y maduración ósea se realiza por un efecto directo sobre los condrocitos en la lámina epifisaria del hueso y que este efecto es potenciado por la hormona de crecimiento ${ }^{14}$. El músculo esquelético también está influido por las hormonas tiroideas, por lo que descensos de sus niveles disminuyen el vigor muscular, afectando consecuentemente el desarrollo óseo.

Luego de la confirmación diagnóstica del estado de hipotiroidismo que afectaba a una majada de la Provincia de Formosa ${ }^{25}$, en nuestro grupo de trabajo surgió el interés de evaluar cómo incidía esta patología sobre el crecimiento y desarrollo de cabritos recientemente destetados, mediante la evolución comparativa del peso vivo, condición corporal y medidas morfométricas.

\section{MATERIAL Y MÉTODOS}

Los cabritos provinieron de un establecimiento cercano a la localidad de Ibarreta (centro de la provincia de Formosa, a $200 \mathrm{~km}$ al oeste de su ciudad capital), donde se efectúa casi exclusivamente la caprinocultura de tipo familiar. Se muestrearon animales de ambos sexos destetados recientemente, de tres meses de edad, mestizos provenientes de cruzamientos de razas Anglo Nubia, criolla y Boer. Recibían un manejo tradicional de tipo extensivo, alimentados exclusivamente sobre pasturas y bosques bajos naturales durante 8 a 10 horas diarias. Estos vegetales fueron muestreados para su análisis químico y procesados por técnicas estandarizadas por la $\mathrm{AOAC}^{1}$ (Tabla 1).

Se tomaron diez muestras de aproximadamente $200 \mathrm{~g}$ de pasto, simulando con la mano la selección que hace el animal en pastoreo; en el caso de los arbustos se 
Tabla 1. Composición química del alimento que disponía la majada (promedios).

\begin{tabular}{lcc}
\hline variable & pastos & arbustos \\
\hline MS (\%) & 22,45 & 40,53 \\
cenizas (\% de MS) & 4,80 & 6,56 \\
$\mathrm{Ca}(\%$ de MS $)$ & 0,25 & 1,02 \\
$\mathrm{Mg}(\%$ de MS) & 0,28 & 1,25 \\
$\mathrm{P}(\%$ de MS) & 0,28 & 0,20 \\
$\mathrm{Fe}(\mathrm{mg} / \mathrm{kg})$ & 107 & 105 \\
$\mathrm{Cu}(\mathrm{mg} / \mathrm{kg})$ & 4,4 & 6,5 \\
$\mathrm{Zn}(\mathrm{mg} / \mathrm{kg})$ & 12,8 & 39 \\
\hline
\end{tabular}

Tabla 2. Minerales séricos en cabritos de ambos gru$\operatorname{pos}(\overline{\mathrm{X}} \pm \mathrm{DE})$.

\begin{tabular}{lcc}
\hline minerales & $\begin{array}{c}\text { hipotiroideos } \\
(\mathrm{n}=10)\end{array}$ & $\begin{array}{c}\text { eutiroideos } \\
(\mathrm{n}=10)\end{array}$ \\
\hline $\mathrm{Ca}(\mathrm{g} / \mathrm{dl}))$ & $8,17 \pm 0,69$ & $8,16 \pm 0,51$ \\
$\mathrm{Mg}(\mathrm{g} / \mathrm{dl})$ & $1,98 \pm 0,33$ & $1,80 \pm 0,10$ \\
$\mathrm{P}(\mathrm{g} / \mathrm{dl})$ & $4,78 \pm 0,88$ & $4,71 \pm 0,69$ \\
$\mathrm{Fe}(\mathrm{ug} / \mathrm{dl})$ & $103,70 \pm 38,18$ & $109,90 \pm 13,6$ \\
$\mathrm{Cu}(\mathrm{ug} / \mathrm{dl})$ & $82,80 \pm 12,68$ & $97,11 \pm 16,69$ \\
$\mathrm{Zn}(\mathrm{ug} / \mathrm{dl})$ & $80,60 \pm 12,50$ & $86,90 \pm 12,33$ \\
\hline
\end{tabular}

No se registraron diferencias significativas entre grupos.

simuló el ramoneo que realizan según su altura de consumo ${ }^{19}$, aplicándose una técnica selectiva de las partes más tiernas. Las especies halladas fueron variadas; dentro del estrato herbáceo se encontraron gramíneas de los géneros Setaria, Gounia, Digitaria, Gymnopogon, Panicum y Chloris y en el estrato arbustivo varias especies de Schimus, Valleria glabra, Prosopis ruscifolia (vinal) y Geoffroea decorticans (chañar), entre otras.

Se trabajó con dos grupos de 10 animales cada uno que fueron individualizados mediante caravanas y seleccionados por presentar o no signos clínicos de bocio; estos últimos fueron considerados como controles de referencia. Con el fin de minimizar los efectos edad y sexo, se los seleccionó a todos de la misma edad (tres meses) y considerando igual cantidad de machos y hembras dentro de cada grupo.

La evaluación de la glándula tiroides en los dos grupos de animales fue realizada a través de dosajes plasmáticos de hormonas $\mathrm{T}_{3}, \mathrm{~T}_{4}$ basal y $\mathrm{T}_{4}$ a los 120 minutos post inyección de hormona liberadora de tirotropina-TRH (TRHelea ${ }^{\circledR}$ ) a dosis de $1 \mathrm{ug} / \mathrm{kg}$ vía endovenosa; lo cual permitió confirmar el estado de hipotiroidismo que aquejaba al lote con bocio ${ }^{25}$. También se analizó en ambos grupos el contenido de macro y microminerales séricos por espectrofotometría de absorción atómica y molecular (Tabla 2).

Para evaluar el crecimiento y desarrollo corporal de los cabritos se procedió a determinar a los 0, 30, 60 y 90 días posteriores, las siguientes variables dependientes:

Peso corporal: determinado mediante balanza electrónica digital marca Dixier Kretz, con una capacidad máxima de $200 \mathrm{~kg}$ y un error de $\pm 0,1 \mathrm{~kg}$.
Condición corporal: valorada según escala del 1 a 5 propuesta por algunos autores ${ }^{9}$.

Valores morfoestructurales: mediante una cinta métrica rígida y otra flexible se tomaron las medidas propuestas en la bibliografía 4, 16 (Figura 1): alzada a la cruz (ACR), diámetro longitudinal (DL), perímetro torácico $(\mathrm{PT})$, diámetro dorsoesternal (DE), anchura de grupa (AG) y perímetro de caña anterior (PC).

Índices morfoestructurales fueron calculados utilizando los valores antes descriptos:

Índice corporal $(\mathrm{ICO})=\mathrm{DL} \times 100 / \mathrm{PT}$.

Índice de proporcionalidad $(\mathrm{IPRO})=\mathrm{DL} \times 100 /$ ACR.

Índice metacarpotorácico $(\mathrm{IMETO})=\mathrm{PC} \times 100 / \mathrm{PT}$. Índice de profundidad relativa de tórax $(\mathrm{IPRP})=$ DE x 100/ACR.

Índice pelviano transversal $($ IPET) $=$ AG $\times 100 /$ ACR.

Para el análisis estadístico de los datos, los valores obtenidos se tabularon categóricamente en planillas de cálculo (Excel) y se procesaron mediante el software Infostat ${ }^{18}$. La normalidad distributiva de cada variable dependiente (peso corporal, condición corporal, medidas e índices morfoestructurales) se verificó a través del test de Wilk-Shapiro, lo que permitió el uso de estadísticas paramétricas de tendencia central (media aritmética) y dispersión (desvío estándar). El grado de asociación lineal se constató por correlación de Pearson. Para las variables dependientes se utilizó un diseño de medidas repetidas completo al azar y un análisis de la variancia (ANOVA) a dos vías. La homogeneidad de la variancia se indagó por el test de Bartlett. Para todas las inferencias se estipuló $\alpha=5 \%$, por debajo del cual se rechazó la hipótesis nula de igualdad entre grupos.

\section{RESULTADOS}

Entre los grupos analizados (eutiroideos e hipotiroideos) no se encontraron diferencias significativas respecto a la ganancia de peso total medida cada 30 días ni en la ganancia de peso diaria calculada (Tabla 3).

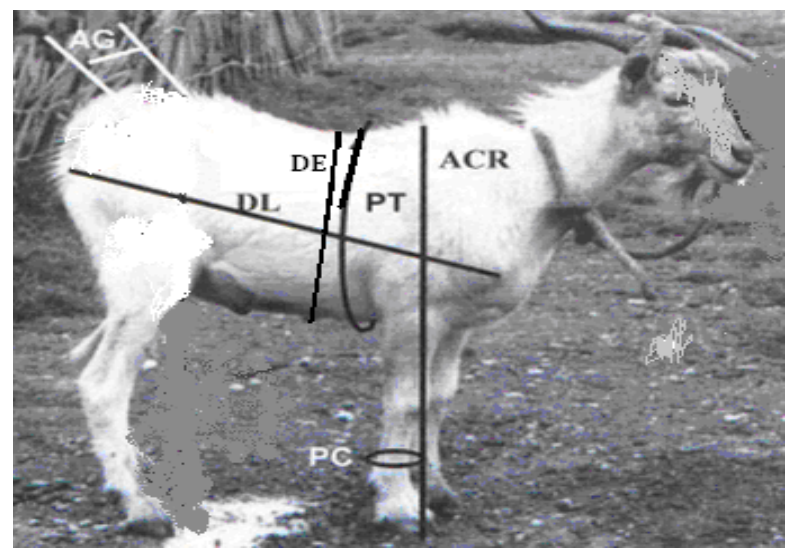

Figura 1. Puntos de referencia para la medición corporal y determinación de los parámetros morfoestructurales en caprinos. In: Hernández Zepeda et al. (2002). 
Tabla 3. Ganancia de peso vivo y medidas morfométricas en ambos grupos $(\overline{\mathrm{x}} \pm \mathrm{DE})$.

\begin{tabular}{|c|c|c|c|c|c|c|c|c|}
\hline \multirow{2}{*}{$\mathrm{V}$} & \multicolumn{2}{|c|}{ día 0} & \multicolumn{2}{|c|}{ día 30} & \multicolumn{2}{|c|}{ día 60} & \multicolumn{2}{|c|}{ día 90} \\
\hline & $\mathrm{H}$ & $\mathrm{E}$ & $\mathrm{H}$ & E & $\mathrm{H}$ & E & $\mathrm{H}$ & E \\
\hline PV (kg) & $\begin{array}{l}11,05 \\
\pm 2,23\end{array}$ & $\begin{array}{l}11,84 \\
\pm 1,96\end{array}$ & $\begin{array}{l}12,75 \\
\pm 2,89\end{array}$ & $\begin{array}{l}13,47 \\
\pm 1,78\end{array}$ & $\begin{array}{l}15,65 \\
\pm 3,48\end{array}$ & $\begin{array}{l}15,45 \\
\pm 2,14\end{array}$ & $\begin{array}{l}19,12 \\
\pm 3,92\end{array}$ & $\begin{array}{c}18,98 \\
\pm 2,8\end{array}$ \\
\hline GPV (kg) & - & - & $\begin{array}{c}1,70 \\
\pm 0,84\end{array}$ & $\begin{array}{c}1,63 \\
\pm 0,78\end{array}$ & $\begin{array}{l}2,90 \\
\pm 1,01\end{array}$ & $\begin{array}{l}1,98 \\
\pm 1,21\end{array}$ & $\begin{array}{c}3,47 \\
\pm 0,71\end{array}$ & $\begin{array}{c}3,53 \\
\pm 0,36\end{array}$ \\
\hline CC (score) & $\begin{array}{c}2 \\
\pm 0,1\end{array}$ & $\begin{array}{c}2 \\
\pm 0,1\end{array}$ & $\begin{array}{c}2 \\
\pm 0,1\end{array}$ & $\begin{array}{c}2,25 \\
\pm 0,46\end{array}$ & $\begin{array}{c}2,43 \\
\pm 0,53\end{array}$ & $\begin{array}{c}2,14 \\
\pm 0,69\end{array}$ & $\begin{array}{c}2,25 \\
\pm 0,82\end{array}$ & $\begin{array}{c}2,27 \\
\pm 0,52\end{array}$ \\
\hline $\mathrm{ACR}(\mathrm{cm})$ & $\begin{array}{c}44,42 \\
\pm 3,80 \mathrm{a}\end{array}$ & $\begin{array}{c}50,08 \\
\pm 4,54 \mathrm{~b}\end{array}$ & $\begin{array}{c}46,25 \\
\pm 3,91 \mathrm{a}\end{array}$ & $\begin{array}{c}52,92 \\
\pm 3,38 \mathrm{~b}\end{array}$ & $\begin{array}{c}48,09 \\
\pm 3,09 \mathrm{a}\end{array}$ & $\begin{array}{c}54,93 \\
\pm 3,39 \mathrm{~b}\end{array}$ & $\begin{array}{c}51,55 \\
\pm 3,09 \mathrm{a}\end{array}$ & $\begin{array}{c}56,93 \\
\pm 2,12 \mathrm{~b}\end{array}$ \\
\hline $\mathrm{DL}(\mathrm{cm})$ & $\begin{array}{l}48,67 \\
\pm 5,75\end{array}$ & $\begin{array}{l}49,00 \\
\pm 3,79\end{array}$ & $\begin{array}{l}50,83 \\
\pm 5,85\end{array}$ & $\begin{array}{l}51,33 \\
\pm 2,25\end{array}$ & $\begin{array}{l}53,00 \\
\pm 4,78\end{array}$ & $\begin{array}{l}51,58 \\
\pm 2,25\end{array}$ & $\begin{array}{c}54,5 \\
\pm 3,94\end{array}$ & $\begin{array}{l}53,08 \\
\pm 2,65\end{array}$ \\
\hline PT (cm) & $\begin{array}{l}54,83 \\
\pm 5,49\end{array}$ & $\begin{array}{l}56,33 \\
\pm 2,58\end{array}$ & $\begin{array}{l}55,83 \\
\pm 4,92\end{array}$ & $\begin{array}{l}56,83 \\
\pm 2,93\end{array}$ & $\begin{array}{l}59,58 \\
\pm 2,84\end{array}$ & $\begin{array}{l}59,92 \\
\pm 3,93\end{array}$ & $\begin{array}{l}61,17 \\
\pm 2,48\end{array}$ & $\begin{array}{l}61,75 \\
\pm 3,83\end{array}$ \\
\hline $\mathrm{DE}(\mathrm{cm})$ & $\begin{array}{l}26,17 \\
\pm 2,32\end{array}$ & $\begin{array}{l}27,83 \\
\pm 2,23\end{array}$ & $\begin{array}{c}28,5 \\
\pm 2,07\end{array}$ & $\begin{array}{c}29 \\
\pm 2,53\end{array}$ & $\begin{array}{l}29,75 \\
\pm 1,78\end{array}$ & $\begin{array}{l}30,25 \\
\pm 1,89\end{array}$ & $\begin{array}{l}31,25 \\
\pm 1,25\end{array}$ & $\begin{array}{c}32,0 \\
\pm 1,55\end{array}$ \\
\hline $\mathrm{AG}(\mathrm{cm})$ & $\begin{array}{c}9,58 \\
\pm 1,11\end{array}$ & $\begin{array}{l}9,08 \\
\pm 0,8\end{array}$ & $\begin{array}{c}9,83 \\
\pm 0,99\end{array}$ & $\begin{array}{c}9,67 \\
\pm 0,52\end{array}$ & $\begin{array}{l}10,08 \\
\pm 1,07\end{array}$ & $\begin{array}{c}9,83 \\
\pm 0,75\end{array}$ & $\begin{array}{l}10,33 \\
\pm 0,75\end{array}$ & $\begin{array}{c}10,5 \\
\pm 0,63\end{array}$ \\
\hline $\mathrm{PC}(\mathrm{cm})$ & $\begin{array}{c}6,95 \\
\pm 0,42\end{array}$ & $\begin{array}{c}6,67 \\
\pm 0,41\end{array}$ & $\begin{array}{c}7,5 \\
\pm 0,55\end{array}$ & $\begin{array}{c}7,08 \\
\pm 0,20\end{array}$ & $\begin{array}{c}7,58 \\
\pm 0,49\end{array}$ & $\begin{array}{c}7,33 \\
\pm 0,42\end{array}$ & $\begin{array}{c}7,75 \\
\pm 0,42\end{array}$ & $\begin{array}{c}7,92 \\
\pm 0,35\end{array}$ \\
\hline
\end{tabular}

H: hipotiroideo, E: eutiroideo, V: variable, PV: peso vivo, GPV: ganancia de PV, CC: condición corporal, ACR: alzada a la cruz, DL: diámetro longitudinal, PT: perímetro torácico, DE: diámetro dorsoesternal, AG: ancho de grupa, PC: perímetro de caña. En cada línea letras distintas indican diferencias significativas.

Tabla 4. Valores obtenidos para los índices morfométricos en ambos grupos $(\overline{\mathrm{X}} \pm \mathrm{DE})$.

\begin{tabular}{lcccccccc}
\hline \multirow{2}{*}{ índice } & \multicolumn{2}{c}{ día 0} & \multicolumn{2}{c}{ día 30} & \multicolumn{2}{c}{ día 60} & \multicolumn{2}{c}{ día 90} \\
& $\mathrm{H}$ & $\mathrm{E}$ & $\mathrm{H}$ & $\mathrm{E}$ & $\mathrm{H}$ & $\mathrm{E}$ & $\mathrm{H}$ & $\mathrm{E}$ \\
\hline \multirow{2}{*}{$\mathrm{ICO}$} & 88,89 & 86,99 & 91,31 & 90,38 & 88,85 & 86,3 & 89,05 & 86,06 \\
& $\pm 8,7$ & $\pm 5,79$ & $\pm 9,7$ & $\pm 2,84$ & $\pm 5,7$ & $\pm 4,91$ & $\pm 4,5$ & $\pm 3,06$ \\
\multirow{2}{*}{ IPRO } & 109,67 & 98,26 & 109,7 & 97,4 & 106,88 & 94,23 & 105,31 & 93,50 \\
& $\pm 6,11 \mathrm{a}$ & $\pm 3,79 \mathrm{~b}$ & $\pm 2,6 \mathrm{a}$ & $\pm 4,18 \mathrm{~b}$ & $\pm 2,10 \mathrm{a}$ & $\pm 3,75 \mathrm{~b}$ & $\pm 2,33 \mathrm{a}$ & $\pm 2,81 \mathrm{~b}$ \\
IMET & 12,74 & 11,86 & 13,5 & 12,47 & 12,72 & 12,29 & 12,66 & 12,86 \\
& $\pm 0,96$ & $\pm 0,85$ & $\pm 1,3$ & $\pm 0,82$ & $\pm 0,69$ & $\pm 0,33$ & $\pm 0,23$ & $\pm 0,94$ \\
IPRP & 58,95 & 55,24 & 60,32 & 54,86 & 60,07 & 55,13 & 60,48 & 56,26 \\
& $\pm 1,54 \mathrm{a}$ & $\pm 0,70 \mathrm{~b}$ & $\pm 1,62 \mathrm{a}$ & $\pm 2,23 \mathrm{~b}$ & $\pm 1,38 \mathrm{a}$ & $\pm 1,23 \mathrm{~b}$ & $\pm 1,37 \mathrm{a}$ & $\pm 0,92 \mathrm{~b}$ \\
IPET & 21,63 & 18,29 & 21,33 & 18,34 & 20,40 & 18,02 & 19,98 & 18,49 \\
& $\pm 1,25 \mathrm{a}$ & $\pm 0,86 \mathrm{~b}$ & $\pm 1,16 \mathrm{a}$ & $\pm 0,87 \mathrm{~b}$ & $\pm 0,91 \mathrm{a}$ & $\pm 0,87 \mathrm{~b}$ & $\pm 0,65 \mathrm{a}$ & $\pm 0,75 \mathrm{~b}$ \\
\hline
\end{tabular}

H: hipotiroideo, E: eutiroideo, índices: ICO: corporal, IPRO: de proporcionalidad, IMET: metacarpotorácico, IPRP: profundidad relativa de tórax, IPET: pelviano transversal. En cada línea letras distintas indican diferencias significativas.

También fueron calculados los índices morfoestructurales, obteniéndose luego su correspondiente significación estadística (Tabla 4).

En los índices IPRO, IPRP e IPET se obtuvieron diferencias significativas $(\mathrm{p}<0,05)$; para el cálculo de todos ellos interviene la variable ACR en el denominador, razón por la cual estos índices resultaron significativamente mayores (menor talla) en los animales hipotiroideos.

$\mathrm{Al}$ realizar las matrices de correlación entre las variables que mostraron diferencias significativas (ACR, IPRO, IPRP e IPET) respecto a los niveles de hormonas $\mathrm{T}_{3} \mathrm{y} \mathrm{T}_{4}$, se hallaron elevados coeficientes de correlación tanto positivos $(0,70$ para ACR) como negativos $(-0,66$ para IPRO, $-0,75$ para IPRP y $-0,71$ para IPET). Cabe destacar, que estos coeficientes fueron obtenidos a par- tir de valores con alto grado de significancia en todos los casos $(p<0,005)$.

\section{DISCUSIÓN}

Considerando el contenido mineral de pastura y arbustos consumidos por estos animales, se estarían cubriendo los requerimientos mínimos para los macroelementos fósforo, calcio y magnesio, según lo recomendado por el NRC ${ }^{23}$. Respecto a los micronutrientes, el cobre superó los 4 ppm sugerido como valor crítico, el zinc fue deficiente en los pastos (menor a 20 ppm según algunos autores ${ }^{21}$ ) y el hierro se mostró dentro de los rangos normales (de 50 a 500 ppm). Los valores aquí registrados son coincidentes con estudios realizados en nuestra zona ${ }^{3}$. 
Los perfiles minerales en sangre de ambos grupos de caprinos no presentaron diferencias significativas y sus valores son considerados como fisiológicos ${ }^{15}$. En la zona de estudio ${ }^{3}$ se reportaron algunas deficiencias de minerales en suero de ganado bovino de cría: fósforo en un $44 \%$, calcio en un $30 \%$, cobre en un $50 \%$, zinc en un $30 \%$ y selenio en un $48 \%$ de los animales estudiados, no encontrándose limitantes en las concentraciones de magnesio, hierro, manganeso y potasio.

$\mathrm{Al}$ analizar la ganancia de peso vivo se pudo constatar que no hubieron diferencias significativas entre ambos grupos, resultando valores similares a los hallados anteriormente en nuestra zona durante los primeros 90 días de recría de cabritos criollos y sus cruzas con razas Boer y Anglo Nubian, en el Centro de Validación de Tecnologías Agropecuarias de la Provincia de Formosa (CEDEVA) ${ }^{26}$. Estos valores también son coincidentes con estudios realizados en México ${ }^{17}$ sobre ganancia de peso vivo de cabritos criollos desarrollados en sistemas extensivos, con otros realizados en el noroeste argentino ${ }^{7} \mathrm{y}$ en Brasil ${ }^{24}$ trabajando en la suplementación de cabritos de raza Saanen.

Considerando la evolución de los pesos de los cabritos hipo y eutiroideos no se encontraron diferencias significativas; esto podría explicarse porque la deficiencia hormonal provocaría dos efectos antagónicos que eventualmente podrían compensarse y así evitar diferencias significativas en el breve lapso que duró el estudio. Esto es que por un lado el hipotiroidismo si bien disminuye el crecimiento y desarrollo de los tejidos en general y del osteoartromuscular en particular, por el otro al disminuir el metabolismo basal provoca un enlentecimiento metabólico que favorece un incremento de peso sin aumento en la ingestión de alimentos, acumulación de líquidos por exceso de mucopolisacáridos, lo cual conlleva a que se puedan observar animales hinchados y con una mayor deposición de grasa subcutánea ${ }^{14}$.

Los dos efectos contrapuestos han sido estudiados por otros autores ${ }^{27}$, quienes experimentaron con cabras a partir de un modelo que incluía ingestión de plantas cianogénicas, productoras de bocio por formación de tiocianato a partir de los altos tenores de cianuro que presentaban. Así, lograron diferencias significativas en la ganancia de peso y en el peso de carcasa sólo en el grupo que recibió cianuro de potasio en muy altas dosis $(3 \mathrm{mg} / \mathrm{kg} /$ día de $\mathrm{KCN})$, explicándose la disminución del peso por los cambios sobre el metabolismo lipídico-proteico inducido por la ingestión del tóxico, con una mayor deposición de grasa en el tejido subcutáneo y un menor desarrollo muscular; esto último por el uso de aminoácidos sulfurados en la detoxificación del cianuro. También relacionaron los efectos a los bajos niveles de $\mathrm{T}_{3}$ que se produjeron, los cuales habrían actuado negativamente sobre la secreción de hormona de crecimiento.

Otros investigadores, considerando este efecto contrario y sabiendo que el estado hipotiroideo produce disminución de la generación de calor y menor tasa de oxígeno consumido por unidad de área corporal, indujeron el estado hipotiroideo por ingestión diaria de propiltiouracilo (PTU), logrando así un aumento significativo en la ganancia de peso en el grupo tratado (vacas maduras y novillos en crecimiento), aunque sin cambios evidentes en la recuperación de la capacidad reproductiva, que era buscada como último objetivo de ese trabajo ${ }^{28}$.

En bovinos tratados con distintos niveles y compuestos de selenio suplementario, encontraron que los tratamientos no afectaron los pesos de las vacas ni de sus terneros recién nacidos, mientras que sí observaron diferencias significativas en los niveles de hormonas $T_{3}$, $\mathrm{T}_{4}$ e inmunoglobulinas en sangre ${ }^{2}$. Esto es coincidente con los hallazgos del presente trabajo ya que en instancias previas al estudio se determinaron diferencias significativas en los niveles plasmáticos de ambas hormonas, pero no existieron diferencias en el peso corporal de los animales afectados respecto al grupo control.

Considerando las diferentes variables zoométricas que se estudiaron, sólo puede destacarse la diferencia significativa en los valores de alzada a la cruz (ACR), resultando el grupo hipotiroideo con valores menores respecto al control. Ello sugiere que la influencia del estado hipotiroideo incide principalmente sobre el desarrollo longitudinal de la estructura ósea y cartilaginosa. Esta observación es coincidente con lo registrado en numerosos estudios sobre el efecto de las hormonas tiroideas sobre el desarrollo y crecimiento de diversos mamíferos, pero considerando este parámetro en forma global. Por ejemplo, en potros recién nacidos con bocio hiperplástico se ha reportado retardos del crecimiento, debilidad del esqueleto y de la piel y sus anexos ${ }^{12}$.

Otros trabajos destacan el efecto permisivo sobre el desarrollo del tejido óseo y muscular de estas hormonas al actuar controlando la actividad mitocondrial, y por ende el aporte de ATP a los procesos anabólicos ${ }^{20}$. Convalidando este hallazgo se pudo demostrar que los niveles de $\mathrm{T}_{3}$ en hembras bovinas que están iniciando su fase reproductiva, se incrementan en forma significativa con el peso vivo del animal ${ }^{10}$.

Considerando los resultados del presente estudio, se puede destacar fundamentalmente la influencia de la patología tiroidea sobre el desarrollo longitudinal del hueso, ya que los otros valores zoométricos no arrojaron diferencias significativas. Esto se puede explicar por la acción que tiene la triiodotironina en particular sobre los condrocitos de la lámina epifisaria ósea, favorecida por adecuados niveles de hormona de crecimiento, factores de crecimiento semejantes a la insulina (IGF) y somatomedina ${ }^{8}$.

Esta acción de la hormona $T_{3}$ fue demostrada en una investigación en la cual se incubaba cartílago de pelvis de pollo con combinaciones de IGF I, $\mathrm{T}_{3} \mathrm{y}$ anticuerpos anti IGF I, encontrándose que IGF y $\mathrm{T}_{3}$ tuvieron un efecto similar al producir aumento de peso, proteínas y número de células en el tejido ${ }^{6}$. La hormona $\mathrm{T}_{3}$ produjo además una hipertrofia celular y vacuolas en el citoplasma, algo similar a lo que ocurre en los condroci- 
tos de la zona hipertrófica del cartílago en crecimiento. Al añadir el anticuerpo anti IGF se inhibió el aumento de peso pero no la hipertrofia celular, lo que sugiere que las hormonas tiroideas per se pueden estimular el crecimiento del cartílago, en ausencia de factores de crecimiento y de somatotrofina. Además, estudios clínicos realizados en seres humanos han confirmado la interrelación entre la disfunción tiroidea y los IGFs, ya que en niños y adolescentes con hipotiroidismo disminuyen los valores de IGF mientras que éstos aumentan en respuesta al tratamiento con levotiroxina.

Se concluye que el estado hipotiroideo afecta el crecimiento de cabritos jóvenes provocando disminución de valores de las variables zoométricas relacionadas al desarrollo longitudinal del hueso y cartílago, provocando una menor talla del animal, lo cual puede implicar consecuencias adversas en el sistema productivo caprino.

\section{REFERENCIAS}

1. Association of Official Agricultural Chemistry (AOAC). 1970. Official methods of analysis, $11^{\mathrm{a}} \mathrm{ed}, \mathrm{Ed}$ AOAC, Washington, p. 1015.

2. Awadeh F, Kincaid R, Johnson K. 1998. Effect of level and source of dietary selenium on concentrations of thyroid hormones and immunoglobulins in beef cows and calves. J Anim Sci 76: 1204-1215.

3. Balbuena O, Mc Dowell L, Toledo H, Conrad J, Wilkinson N, Martin F. 1989. Estudios de la nutrición mineral de los bovinos para carne del este de las provincias de Chaco y Formosa (Argentina). Vet Arg 6: 584-594.

4. Bedotti D, Gómez M, Sánchez N, Martos Peinado J. 2004. Caracterización morfológica y faneróptica de la cabra colorada pampeana. Arch Zoot 53: 261-271.

5. Boza J, Robles A, Fernández P, Bermudez F. 1997. Planificación ganadera de pastos de zonas desfavorecidas. Arch Zootec 51: 341-349.

6. Buch WM, Van Wyk JJ. 1987. Triiodothyronine stimulates cartilage growth and maturation by different mechanism. Am J Physiol 252: E178-E182.

7. Chagra P, Leguiza D, Vera T. 2008. Factores que inciden en el crecimiento de los cabritos lechales y la producción de leche invernal de cabras criollas biotipo regional. Informe INTA La Rioja. On line: http://www.inta.gov.ar/larioja/info/indices/tematica/ganaderia/ganad_caprina.htm.

8. Chernausek SD, Underwood LE, Utiger RD. 1983. Growth hormone secretion and plasma somatomedin C in hypothyroidism. Oxford Clin Endocrinol 19: 337-44.

9. Coffey L, Hale M, Wells A. 2004. Goats: sustainable production overview. On line: http://www.attra.ncat.org NCAT.

10. Colmenarez D, Leonardi F, Brevo M. 2008. Niveles de T3 y T4 en bovinos Holstein de 2-24 meses de edad. On line: http://www. pegasus.ucla. edu.ve/ccc/revista.

11. Deza C, Bascur F, Pérez G, Díaz M, Baroglio C. 2003. Identificación de variables morfoestructurales y de polimorfismos sanguíneos para la caracterización de las ca- bras criollas en el NO de Córdoba. Agriscientia (Córdoba, Argentina) 20: 69-77.

12. Doige C, McLaughlin B. 1981. Hyperplastic goitre in newborn foals in western Canada. Can Vet J 22: 42-45.

13. French M. 1975. Observaciones sobre las cabras, Ed FAO, Roma, p. 234.

14. García Sacristán A. 1995. Fisiología Veterinaria, Ed McGraw, Madrid, p. 707-716.

15. Grace ND. 1989. The mineral requirements of grazing ruminants, Ed New Zealand Society of Animal Production, Hamilton (New Zealand), p. 87.

16. Hernández Zepeda J, Franco F, Herrera M, Rodero E, Sierra A, Bañuelos A. 2002. Estudios de los recursos genéticos de México: características morfológicas y morfoestructurales de los caprinos nativos de Puebla. Arch Zootec 51: 53-64.

17. Hernández Zepeda J, Herrera M, Rodero E, Vargas S, Villareal E. 2005. Tendencia en el crecimiento de cabritos criollos en sistemas extensivos. Arch Zootec 54: 429-436.

18. Infostat. 2002. Programa Estadístico Versión 1.1. Grupo Estadística y Diseño, FCA Universidad Nacional de Córdoba, Argentina.

19. Lamela L. 1998. Métodos de muestreo y mediciones en sistemas silvopastoriles. Compendio de conferencias para el diplomado en silvopastoreo, Ed. Mineo, Matanzas (Cuba), p. 12.

20. Matamoros R, Gomez C, Andaur M. 2002. Hormonas de utilidad diagnóstica en medicina veterinaria. Arch Med Vet 34: 167-182.

21. Mayland H, Rosenau R, Florence A. 1980. Grazing cow and calf responses to zinc supplementation. J Animal Sci 51: 966-974.

22. Morales CA, Rodríguez N. 2005. Hormonas tiroideas en la reproducción y en la producción láctea del ganado lechero: revisión de literatura. Rev Col Ciencia Pecuaria 18: 137.

23. National Research Council (NRC). 1984. Nutrient requirements of domestic animals. nutrient requirements of goats: angora, dairy and meat goats in temperate and tropical countries, Publ. N ${ }^{\circ} 15$, National Academy Press, Washington, $75 \mathrm{p}$.

24. Nunes Medeiros A, Germano R, Batista I, Ramos F. 2008. Desarrollo en recría de caprinos de raza Saanen alimentados con dietas completas conteniendo diferentes niveles de heno de pasto elefante. Arch Latinoam Prod Anim 16: $56-63$.

25. Ortiz ML, Brem JJ, Mancebo OA, Trulls HE, Picot JA, Brem JC. 2008. Confirmación diagnóstica de hipotiroidismo en cabras de la Provincia de Formosa, Argentina. Rev Vet 19: 42-45.

26. Revidatti MA, Sánchez S, De La Rosa S, Ayala S. 2008. Ganancia de peso postdestete y mortandad de cabritos en el oeste de Formosa. On line: http//www.unne. edu.ar/web/ cyt/com.2008/index.htm.

27. Soto-Blanco B, Gorniak S, Kimura E. 2001. Physiopathological effects of the administration of chronic cyanide to growing goats - a model of ingestion of cyanogenic plants. Vet Res Com 255: 379-389. 
28. Thrift T, Bernal A, Lewis A, Neuendorff D, Willard C, Randel R. 1999. Effects of induced hypothyroidism on weight gains, lactation and reproductive performance of primiparous Brahman cows. J Anim Sci 77: 1844-1850.
29. Vargas López S, Sánchez M. 2001. La cabra criolla como componente del sistema agrosilvopastoril de subsistencia en Puebla, México. Memorias XXVI Jornadas Cientificas y VInternacionales de la Sociedad Española de Ovinotecnia y Caprinotecnia, Sevilla (España), p. 903-908. 